\title{
Attentional capture with various distractor and target types
}

\author{
GARVIN CHASTAIN \\ Boise State University, Boise, Idaho \\ and \\ MARYLOU CHEAL \\ Arizona State University, Tempe, Arizona
}

\begin{abstract}
Effects of nonpredictive distractors that involved changes in luminance, size, or shape were examined in three experiments. In Experiment 1, with two types of distractors (onsets and offsets), accuracy was better on trials when the distractor was near the location of either an offset or an onset target than on trials when the distractor was in a different location from that of the target, demonstrating attentional capture. Capture occurred both when the type of target (onset or offset) was blocked and therefore predictable and also when the type of target was mixed within blocks and therefore not predictable. Further experiments indicated that distractors captured attention even when the change to distractor did not create a new perceptual object. Neither a singleton-detection mode, nor a contingent involuntary orienting hypothesis, nor creation of a new object seems to explain all of these data adequately. Rather, capture may depend on a number of factors in the task.
\end{abstract}

The topic of capture of attention has a long history in the psychological literature. At this point, it seems obvious that there is no single simple answer as to whether some particular item in the visual field can capture a person's attention if that person has no preconception of what might appear. Although some have recently stated that attentional capture cannot occur without some prior knowledge of the target (Folk, Remington, \& Johnston, 1992; Folk, Remington, \& Wright, 1994; Gibson \& Kelsey, 1998; Hillstrom \& Yantis, 1994; Yantis, 1993; Yantis \& Hillstrom, 1994), intuitively it does not seem possible that this would be the case in all circumstances. If a bee were to sting you, this would no doubt take your attention away from the weeds in the garden even if you had not seen the bee. Considerable past research has supported the idea of stimulus-driven attentional capture (Jonides \& Yantis, 1988; Yantis, 1993; Yantis \& Jonides, 1984). However, in other cases, even though a visual stimulus could pop out of background items, it did not capture attention when the field of items appeared (Chastain, 1996; Chastain \& Cheal, 1998).

An important contradiction to simple capture of attention has been demonstrated in experiments in which the item to capture attention (called a distractor) and a target (presented on successive screens) could be either a unique

The authors are indebted to Chip Folk, Derrick Watson, and Jeremy Wolfe for useful comments on an earlier version of the manuscript. They also thank Shane Clubb, Jennifer Galyen, Renee Harrold, Shelley McFall, Elizabeth Wood, and Natalie Young for assistance in data collection. Correspondence should be addressed to M. Cheal, $127 \mathrm{E}$. Loma Vista Drive, Tempe, AZ 85282-3574(e-mail: cheal@asu.edu). color or a unique stimulus onset. It has been found that colored distractors capture attention when the target is a color, and onset distractors capture attention when the target is an onset, but neither distractor captures attention when the target is the opposite type (Folk et al., 1992; Folk et al., 1994; Gibson \& Kelsey, 1998).

Literature produced over the long history of the locationcuing paradigm has been based on the concept that a bright onset of a light will facilitate attention for targets that after a short interval appear near that location (Cheal \& Lyon, 1989; Eriksen \& Collins, 1969; Eriksen \& Hoffman, 1972a, 1972b, 1973; Holmgren, 1974; Jonides, 1980; Posner, 1980; Sperling \& Melchner, 1978). Further work has supported the idea that certain stimuli do capture attention (Cheal \& Chastain, in press). However, questions as to whether attention is ever drawn automatically by a stimulus challenge that literature. For example, Bacon and Egeth (1994) have suggested that participants develop a singleton-detection mode when the target is a unique character among other characters. In such cases, any singleton (whether appearing as the sole character or as a unique character among other characters) would capture attention.

Folk and colleagues (Folk et al., 1992; Folk et al., 1994) promoted the "contingent involuntary orienting hypothesis." They argued that an abrupt-onset peripheral distractor derives its ability to draw attention to its location because it shares characteristics of the target and because participants are set to attend to those characteristics. Gibson \& Kelsey (1998) extended this hypothesis to suggest that there are screen-wide characteristics of the target screen that lead to the anticipation of the target from a 
prior distractor. In another interpretation, Yantis and Jonides (1984; Jonides \& Yantis, 1988) argue that an abruptonset precue captures attention because it signals the presence of a new object, to which the visual processing system is very sensitive. If the offset of part of a pattern produces a new object, this also could capture attention.

In some recent research, the onset or offset of stimulus characters has been used to study the "contingent involuntary orienting hypothesis." An offset distractor would not appear to share specific characteristics that the participant is seeking in an onset target. However, offsets were found to capture attention (Chastain \& Cheal, 1999; Theeuwes, 1990, 1991). Nevertheless, if the creation of a distractor by an offset that appears on a pretarget screen indicates that the target onset is impending, this may provide a display-wide cue (Gibson \& Kelsey, 1998). Therefore, offsets might attract attention. Atchley, Kramer, and Hillstrom (2000) found that an offset distractor could capture attention but only when all stimuli on the target screen appeared as offsets and not when all target stimuli were onsets.

These data, along with other recent data of participants who optimize responses by adapting a strategy that is most efficient for the conditions of each block of trials (Chastain \& Cheal, 2001), suggest that one simple rule cannot account for all of the data. Obviously, a set can be developed for a singleton (Bacon \& Egeth, 1994). In addition, contingent involuntary orienting can occur when there is a contingency between the target and prior distractors (Folk, Remington, \& Johnston, 1993), and a screen-wide set can occur when the situation allows (Atchley et al., 2000; Gibson \& Kelsey, 1998). However, it is also possible that other conditions can be found in which none of these can apply. If, of course, one wants to say that any change on the target screen can result in a set for any change on the distractor screen, then there can be no argument, because a change must occur for a target screen to appear. This does not seem to be a very useful concept, however, because it does not account for capture by some stimuli and not others when the target does not differ, and presumably, any set would be the same. Differential effects of capture have occurred with different distractors even though the target screen was always the same (Chastain, Cheal, \& Kuskova, in press). In this paper, the effects of specific stimuli upon specific targets will be examined.

Intuitively, it may seem that an abruptly appearing pattern in the visual field would attract attention, whereas a pattern that disappears abruptly would not be as likely to do so. This is because the sudden presence of something may be more important than a sudden absence. However, in visual search an onset has been found to have no more influence on finding the target than an offset (Watson \& Humphreys, 1995) and onsets may not pop out if embedded in offsets of similar luminance change (Miller, 1989). Likewise, attentional capture, in which attention is automatically drawn by a stimulus in the visual field, has been shown with abrupt offsets as well as with abrupt onsets (Atchley et al., 2000; Chastain \& Cheal, 1999). However, the question of whether onsets or offsets can capture attention when the target is of a different type has not been explored thoroughly. Therefore, in the first experiment here, both onset or offset distractors and onset or offset targets were used in order to examine the role of onsets and offsets in attentional capture. Unlike the target screens used by Atchley et al. (2000) for their Experiments 1 and 2, in which all characters were either onsets or offsets on any given trial, those in the present Experiment 1 were always two onset characters and two offset characters. Our strategy was to prevent any screenwide set for either onsets or offsets, although, of course, there were luminance changes from screen to screen on all trials. We then manipulated whether a set could be formed that was dependent on target onset or offset.

For one group in Experiment 1, onset and offset targets were presented in separate blocks, following a screen containing either an offset or an onset distractor pattern (distractors were randomized within blocks of trials). Thus, on every trial there were both onsets and offsets on the target screen, but the target type was defined by whether the target itself was an offset or an onset. In each case, the target could not be identified by any property other than its identity. Capture of attention was inferred if there was a larger proportion of correct trials when the distractor and target were near the same location (valid trials) than when they were in different locations (invalid trials). Thus, the data were analyzed to determine whether validity effects, indicating attentional capture, would be stronger with distractor and targets of the same type (both offsets or both onsets) than if they were of different types (one an offset and one an onset). If distractors and targets of the same type resulted in higher accuracy scores than when they differed, predictions based on attentional set for target type would be supported. If validity effects were significant and approximately equal for all distractor- and target-type combinations, this could be interpreted in consonance with the idea that attention is drawn automatically by the appearance of a new object, independent of attentional set.

For a second group of participants in Experiment 1, target types as well as distractor types were randomized within blocks of trials. This group was used to test whether onsets and offsets could capture attention when there was no opportunity to develop a set for an offset target or an onset target. On these trials, there was no opportunity to learn the distractor type until the distractor appeared, and likewise, the first knowledge of target type occurred when the target appeared. In additional experiments, the new object interpretation was tested through presenting changes from the prescreen to the distractor. These changes did not produce a new object for some of the distractor types in Experiment 2, and only dimmed the character on the fixation screen while maintaining its shape in Experiment 3. 


\section{EXPERIMENT 1}

The aim of Experiment 1 was to test for validity effects with onset or offset targets. If there were larger validity effects when distractors and targets were both onsets or both offsets than when they differed, it would support the proposition that the effectiveness of offset or onset distractors in capturing attention is related to their sharing a characteristic with the targets that participants are seeking. One group of participants viewed offset targets shown in a separate block from onset targets, although onset and offset distractors were mixed within each block. For the other group of participants, onset and offset targets and onset and offset distractors were randomized in the same block of trials.

\section{Method}

Participants. Participants were drawn from the introductory psychology subject pool at Boise State University and received course credit for their participation in a single session of approximately $1 \mathrm{~h}$. No participant served in more than one experiment.
Data from any participant who did not obtain better than chance accuracy overall were not used and that participant was replaced. Each participant reported normal or corrected-to-normal visual acuity. In the blocked group, there were 24 participants (10 men and 14 women, mean age of 20.7 years; range of 18-29). In the mixed group, there also were 24 participants ( 8 men and 16 women, mean age of 25.0 years; range of $18-40$ ).

Apparatus. Stimuli for these experiments were presented on a $60-\mathrm{Hz}$ Magnavox MB7000 17-in. monitor, controlled by an IBM PS/2 Model 30. The P-22 phosphor produced displays that had a visible persistence of no longer than $1 \mathrm{msec}$. Distance of the participants' eyes from the display was maintained at approximately $46 \mathrm{~cm}$ by a Gulf \& Western table-mounted chinrest with head restraint. Eye movements were monitored during each trial by the experimenter with a tripod-mounted box camera connected to a television set. If an eye movement was observed immediately before, during, or immediately after the exposure, the participant was admonished by the experimenter. In addition, the short duration of the distractor to target interval $(100$ or $200 \mathrm{msec}$ ) made eye fixation the best strategy for accurate responses.

Stimuli. For Experiment 1, the fixation screen contained a fixation bar $\left(.09^{\circ}\right.$ tall and $.24^{\circ}$ wide), two plus signs and two figure 8 characters (each $1.24^{\circ}$ tall and $.94^{\circ}$ wide), and two sideways Ts

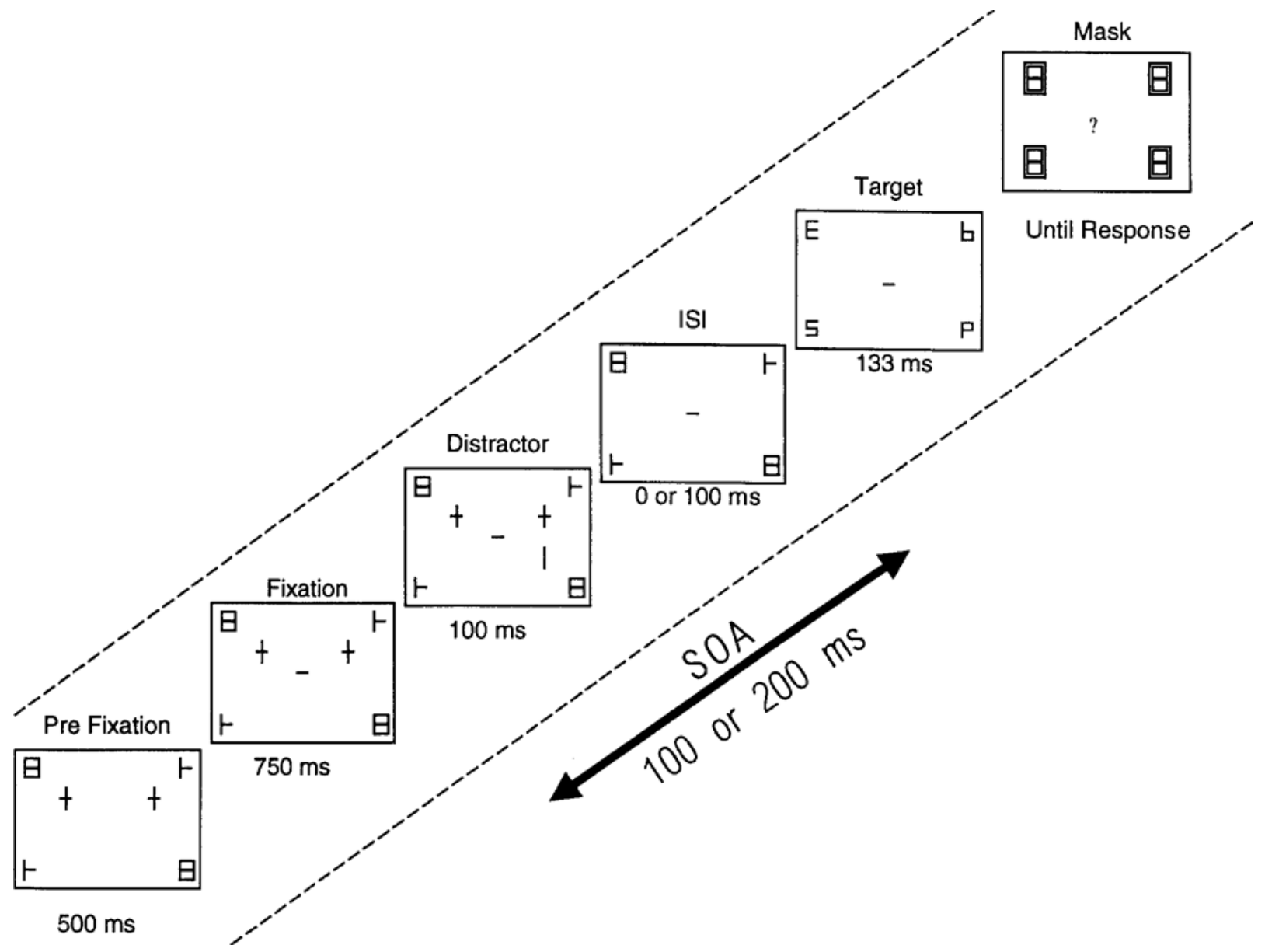

Figure 1. Reverse image of a series of screens for a trial in Experiment 1 (actual stimuli were white on dark gray). After 500 msec, a fixation bar appeared that acted as a warning signal. The plus signs and vertical line disappeared following $100 \mathrm{msec}$ of distractor presentation. The target could be an $\mathrm{E}$ or an $\mathrm{H}$. (Note: Figures 1, 2, and 4 are not drawn to scale.) 
$\left(1.24^{\circ}\right.$ tall and $.72^{\circ}$ wide). The two plus signs were either both above the fixation bar or both below fixation. On a randomly chosen half of the exposures, the $8 \mathrm{~s}$ on the fixation screen were at upper left and lower right, whereas on the other half, they were at upper right and lower left. Right-facing sideways Ts were always in the remaining two locations.

The four possible target locations were arranged as the vertices of a rectangle centered on fixation, with each location approximately $5.25^{\circ}$ from fixation. Distractor locations were approximately $1^{\circ}$ to the inside of each of these locations. See Figure 1 for the arrangement of these characters. The distractor screen was the same as the fixation screen except that either one plus sign became a single vertical line in the same location or a single vertical line was added in another location. The single vertical line $\left(1.24^{\circ}\right.$ tall and $.24^{\circ}$ wide) was the distractor. All stimuli were white (IBM color 7, $113.1 \mathrm{~cd} / \mathrm{m}^{2}$ ). Thus, there were two possible types of distractors. One was a vertical line produced by the offset of the horizontal crossbar of a plus sign, and the other was the same-sized vertical line that appeared where no character had been (an onset distractor is depicted in Figure 1; both types are shown in Figure 2). For offset distractors, the plus sign that did not contain the offset remained on the screen while the vertical line distractor was present, whereas for onset distractors both plus signs remained on the screen during this time. The target displays always contained a $\mathrm{P}$, an $\mathrm{S}$, and a b as nontargets, and an $\mathrm{E}$ or an $\mathrm{H}$ as the target (all as block characters, each $1.24^{\circ}$ tall and $.94^{\circ}$ wide). The $\mathrm{E}$ or $\mathrm{H}$ target could appear either as the offset of lines from a figure 8 or as the onset of lines to a sideways $\mathrm{T}$
(Figure 2). On the distractor screen, the vertical line distractor always was beside either one of the figure 8 s (for offset targets) or one of the sideways Ts (for onset targets). Thus, the vertical line indicated the target location with $50 \%$ validity for the blocked group. However, for the mixed group, the vertical line indicated the target location with $25 \%$ validity because the target could replace any of the four characters (two figure 8s and two sideways Ts) on the fixation/distractor screens with equal probability. Therefore, mixed group participants could not determine from the location of the vertical line distractor whether the target for a given trial was to be an offset or instead an onset.

For offset targets, the distractor could be either an offset, replacing a plus on the fixation screen, or an onset, appearing where no plus had been on the fixation screen; and likewise for onset targets. From trial to trial, the participants in either group did not know whether the distractor would be an offset or an onset, although for the blocked group the participants could develop an attentional set for an offset target (throughout one block) or an onset target (throughout the other block). For the mixed group, the participants could not develop an attentional set for either onset or offset target or distractor. The fixation and target screens were similar to those in Experiment 2 of Watson and Humphreys (1995).

A white postexposure mask, present at each of the locations that contained a character on the target screen on a given exposure, was an outline of a figure 8 .

Procedure. The participant was familiarized with the possible target screens and responses, and was told that location of neither
Fixation

\section{Offset Distractor:}

\section{Onset Distractor:}

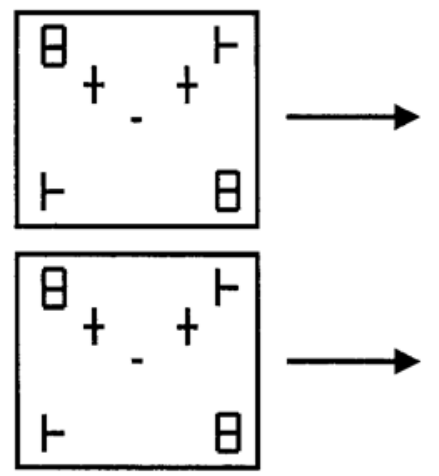

ISI

Offset Target:

\section{Onset Target:}

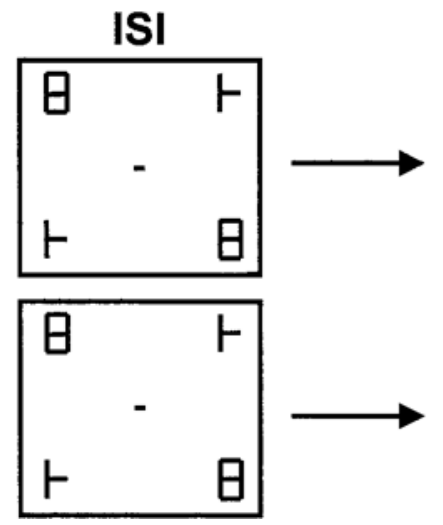

Distractor

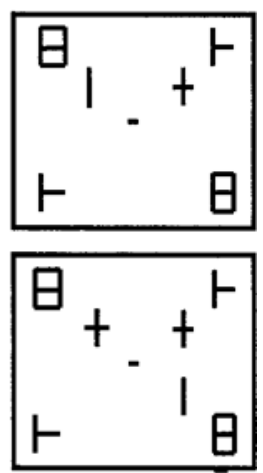

Target

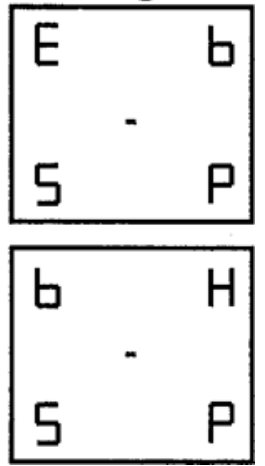

Figure 2. The types of distractors and targets used in Experiment 1. Upper: The same fixation screen could change to an offset distractor or an onset distractor. Lower: The same screen during the interstimulus interval could change to either an offset target (an $E$ is illustrated) or an onset target (an $H$ is illustrated). In either case, the target could be either an $E$ or an $H$. 
the offset among the pluses nor the onset of the vertical would be predictive of the location of the target. The blocked group participants were shown that the vertical line could appear beside the figure 8 (offset target block) or sideways $\mathrm{T}$ (onset target block) that became the target, or beside the other figure 8 or sideways T. The mixed group participants were shown that the vertical line would appear beside a figure 8 or a sideways T, and that any of the two 8s or two sideways Ts would then change to the target with equal probability. Each participant then received 32 practice trials before each block for one group, or an initial 64 practice trials for the other group. The responses for these trials were not recorded.

The participant was first shown a prefixation screen, which did not have a fixation bar. After $500 \mathrm{msec}$, the bar was added. This fixation screen remained for $750 \mathrm{msec}$. The display that contained the distractor immediately followed the fixation screen for $100 \mathrm{msec}$. At this point, the pluses and the distractor were removed and a screen containing only the fixation bar and the four corner characters (two eights and two Ts) appeared for 0 or $100 \mathrm{msec}$ (producing stimulus onset asynchronies [SOAs] of 100 or $200 \mathrm{msec}$ ). Both SOAs used in these experiments were expected to result in facilitation of responding when the distractor and target were near the same location. Two SOAs were used so that participants could not anticipate the onset of the target screen.
At the end of the SOA, the target display appeared for $133 \mathrm{msec}$ for offset targets or $117 \mathrm{msec}$ for onset targets. Different target durations were necessary because pilot work showed that, at the same exposure duration, forward masking made the offset targets slightly less visible than the onset targets. The participant pressed the "up" or "down" arrow on the numeric keypad to indicate "H" or "E," respectively. Accuracy was stressed and reaction time was not recorded. A "happy face" then appeared if the response was correct, or a minus sign if it was incorrect, for $1 \mathrm{sec}$. The next trial began approximately $2 \mathrm{sec}$ later. The participant could pause at any time merely by withholding the response until ready.

Experimental design. For the experimental trials for the blocked group, the two target types were presented in separate blocks, one for offset and the other for onset targets. Half of the participants received the offset first and the other half received the onset first. Each block consisted of three replications of the factorial combination of two distractor types (onset or offset), two distractor validities (valid, invalid), two target locations (above fixation, below fixation), two target identities ( $\mathrm{E}$ or $\mathrm{H}$ ), and two locations of $8 \mathrm{~s}$ on the fixation screen (upper left/lower right; upper right/lower left), and two SOAs (100, $200 \mathrm{msec})$, for 192 trials per block. Variable combinations were presented in a different random order within each block to each participant. For the experimental trials for the
Targets Blocked

? Invalid $\square$ Valid

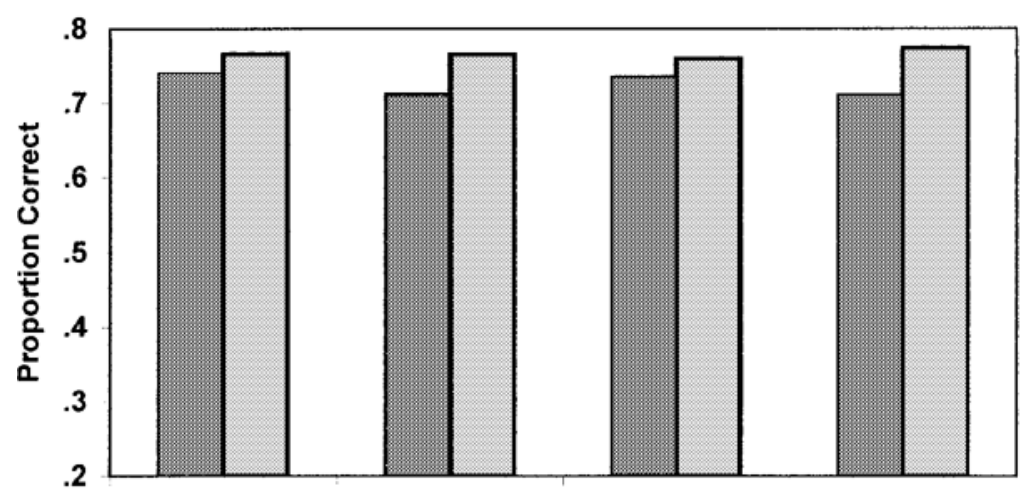

Targets Mixed

[ Invalid $\square$ Valid

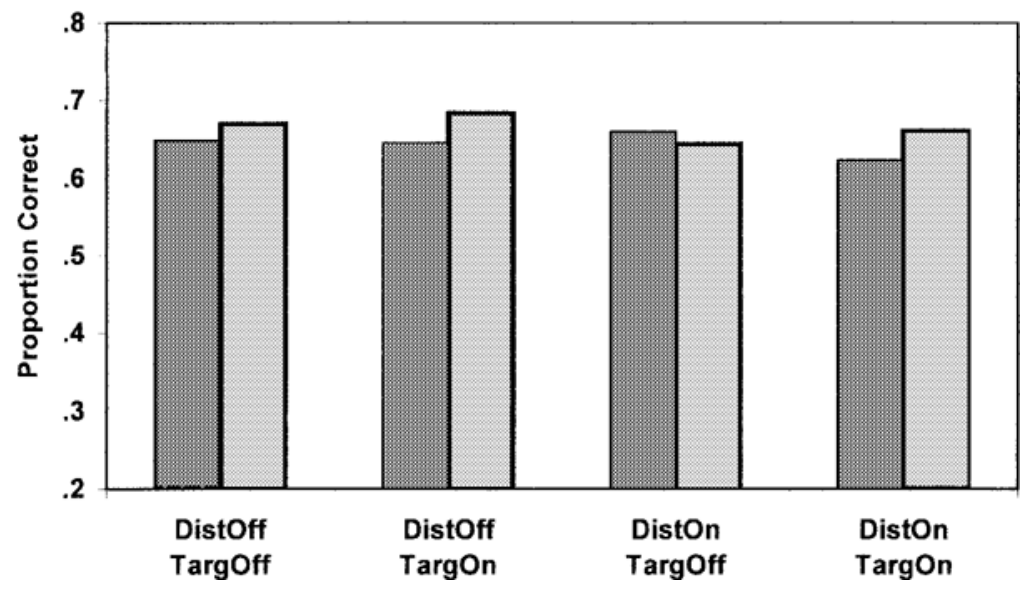

Figure 3. Mean proportion of correct responses for offset (Off) and onset (On) distractor (Dist) and target (Targ) combinations with target type blocked (above) and mixed (below) in Experiment 1. 
mixed group, target types were not blocked; otherwise the design was the same as for the blocked group, with the same total number of trials.

\section{Results and Discussion}

Hi-log linear analyses for dichotomous data were computed on all data. Means for valid and invalid distractors for each distractor and target type combination are presented in Figure 3 for the blocked and mixed groups. Means for all combinations of target type, distractor type, distractor validity, and SOA were entered into an omnibus Hi-log linear analysis, which showed that the only significant effects were three main effects: validity, $\chi^{2}(1)=$ $20.77, p<.0001$ (accuracy was significantly better when the distractor was at the same location as the target than when distractor and target locations differed); blocked versus mixed, $\chi^{2}(1)=157.91, p<.0001$ (accuracy was higher with blocked than with mixed trials), and SOA, $\chi^{2}(1)=$ $29.38, p<.0001$ (accuracy increased with increase in SOA). The effect of SOA was expected; it is of little interest to this research and will not be discussed further.

Accuracy for valid trials was approximately equal in magnitude for onset and offset targets with either onset or offset distractors, particularly for the blocked condition. Therefore, a further test was made to determine whether preknowledge of the target type affected the amount of capture. The data were analyzed as to whether the distractor and target were the same type (either both offsets or both onsets) on a trial or whether they were different (one offset and the other onset). There were significant effects of validity and blocking, but no significant difference as to whether the types were the same or not $\left[\chi^{2}(1)=0.38, p=.54\right]$, and no significant interactions. Thus, blocking of target type, which resulted in preknowledge of the target type, did not affect the amount of capture by the distractor. It is obvious that participants receiving blocked trials used the knowledge that the target could appear in only one of two locations (either replacing one of the $8 \mathrm{~s}$ or replacing one of the Ts, depending on the block), because participants had significantly more correct trials when they were blocked than when they were mixed. The overall lower accuracy of participants in the mixed group indicated a four-location alternative, in contrast to the two-location alternative of participants in the blocked group.

Because targets and nontargets were all different letters, there was no singleton character on the target screen (Figure 2). Therefore, the effect of capture by the vertical line distractor cannot be attributed to a singleton detection strategy (Bacon \& Egeth, 1994). Also, capture was not due to a specific attentional set, because offset distractors produced capture with onset targets and vice versa, and capture occurred with both types of targets mixed with both types of distractors. These results are consistent with those of Chastain and Cheal (1999); an offset distractor captures attention with onset targets. However, Atchley et al. (2000) did not find capture when offset targets appeared with onset distractors or vice versa and target types were blocked in their Experiment 1. An important difference in their method was that all characters on the target screen were either offsets or onsets. Thus, in their Experiment 1 (where the trials were blocked by target type) and Experiment 2 (where trials were mixed but target type was apparent at the beginning of each trial), the participants could have developed a screenwide set for either the offset or the onset of characters. The present experiment differed in that there were both onset and offset characters on the target screen on every trial. Of course, in our blocked group, participants could have developed a set for whether the actual target was an offset or an onset, but there was no screen-wide set for either onset or offset. The finding of capture when distractor and target types do not match was reexamined in the present Experiments 2 and 3.

In addition, the offset of the horizontal line to leave a vertical line as the offset distractor could have produced a new perceptual object, as could the onset of a vertical line for onset distractors. Therefore, Experiment 2 was conducted to eliminate the appearance of a new object as being the basis for attentional capture. This control was accomplished on a third of the trials with a change from one of the pluses to a smaller plus, and on another third of the trials with a change to a plus with a thin vertical line.

\section{EXPERIMENT 2}

In Experiment 1, appearance of the vertical line distractor might have produced a new object that captured attention, whether it appeared as an onset or instead as the disappearance of the horizontal crossbar of a plus sign. The following experiment was conducted to explore this possibility by including conditions in which the lines of the unique distractor item changed relative size while remaining a plus sign (see Figure 4). In these conditions, the plus that was altered still remained a plus, and thus arguably a new object was not produced. If capture occurred with these modifications, this would suggest that the change itself captured attention rather than that a new object produced by the change captured attention. Note that in the three conditions used (change to a vertical line, change to a smaller plus, and change to a different-shaped plus), there was no overall luminance difference in the three types of distractors; all three contained the same number of pixels.

Unlike in Experiment 1, in Experiment 2 the target screen did not vary (except in location of the target). Thus, the distractor screen was varied with the target screen constant for the different distractor conditions. In addition, the character at each potential target location appeared where nothing had been present on the fixation or distractor screen; that is, all targets were onsets, and there were no placeholders at potential target locations as had been the case with the figure $8 \mathrm{~s}$ and sideways $T \mathrm{~s}$ in Experiment 1.

In order to maintain a constant target screen, targets consisted of a single $\mathrm{C}$ in one of four orientations with 
100 or $200 \mathrm{~ms}$

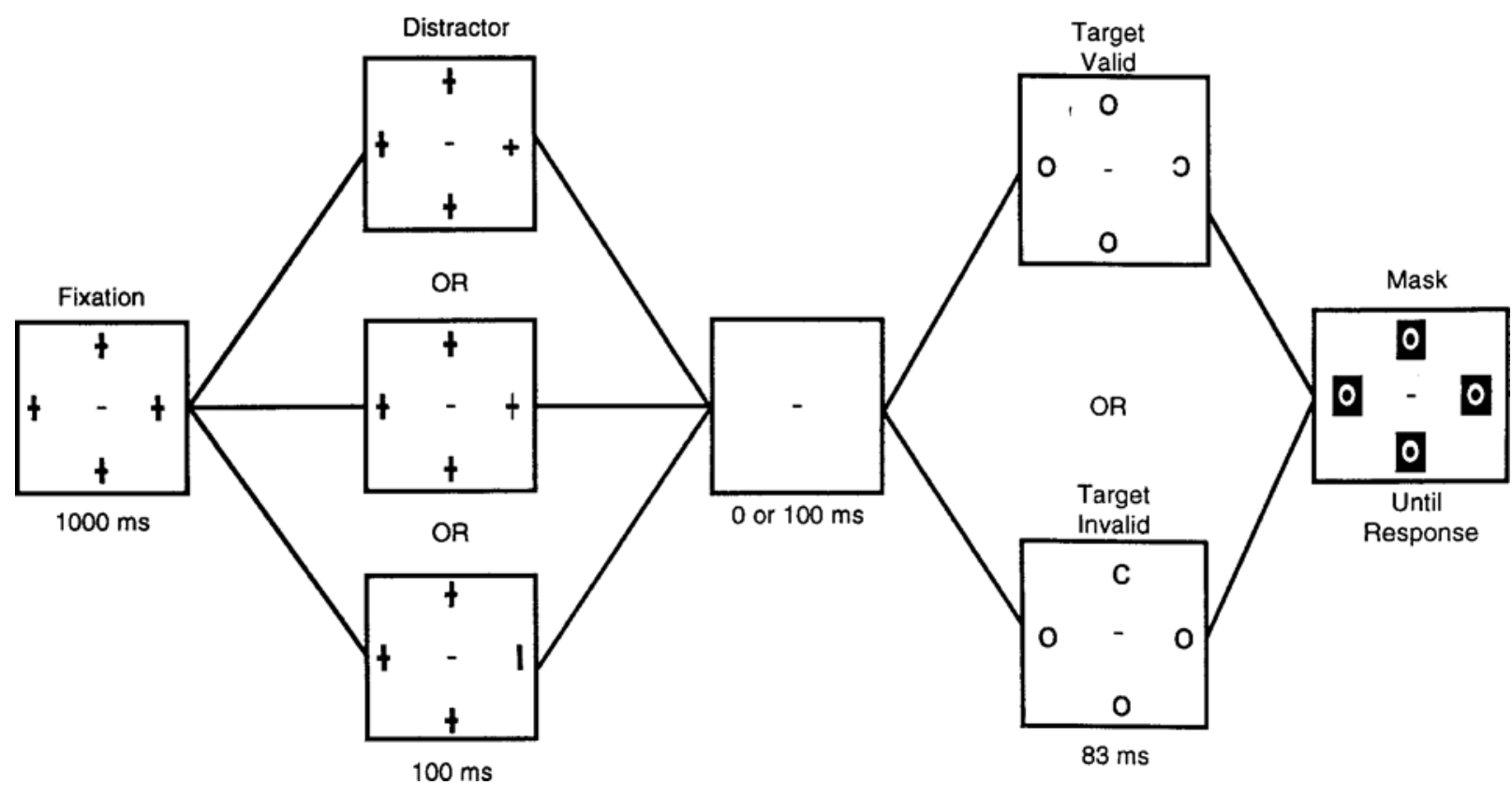

Figure 4. Series of screens for trials in Experiment 2. The three distractor types are illustrated (small plus, plus with skinny vertical line, vertical line). The fixation bar was on throughout trials. The target $\mathrm{C}$ could face in any of four orientations at any one of the four locations.

three complete circles as nontargets. This choice resulted in a singleton $\mathrm{C}$ on the target screen. This aspect of the task was addressed further in Experiment 3.

\section{Method}

Only differences from the method of Experiment 1 will be given here. For Experiment 2, the distractor and target locations were arranged as the vertices of a diamond centered on fixation, with distances approximately the same as in Experiment 1 (Figure 4). There were three groups of 20 participants per group; each group received a different type of distractor. The three types of distractors are depicted in Figure 4. For one group (11 men and 9 women, mean age of 21.2 years; range of 18-31), one of the pluses became a smaller plus sign, which served as the distractor. For another group (7 men and 13 women, mean age of 20.4 years; range of 18-45), one of the plus signs became a plus with a thinner (one pixel wide rather than two pixels wide), but longer, vertical line to serve as the distractor. For the final group ( 7 men and 13 women, mean age of 21.1 years; range of 17-42), the horizontal crossbar of one of the plus signs on the fixation screen offset to produce a vertical line distractor, as in Experiment 1. All distractor types were offsets in the sense that there were fewer pixels in the distractor than in the original plus sign. In order to control luminance as much as possible, after the offset or change to the distractor, each distractor type contained the same number of illuminated pixels (28). The target screen appeared for $83 \mathrm{msec}$; a shorter target duration than in Experiment 1 was sufficient, because there were no placeholders to provide forward masking. The target screen contained four onsets: one white $\mathrm{C}$, with the gap at the top, bottom, left, or right, and three Os (of the same brightness and subtending approximately the same visual angle as did the $\mathrm{C}$ ). The gap in the target was equally likely to be in each of the four orientations. The participant pressed one of the four arrow keys to indicate the orientation of the gap in the target "C." The distractor appeared near the target location only by chance-that is, on $25 \%$ of exposures. There were 3 repetitions of 4 target locations $\times 4$ distractor locations $\times 4$ target identities $\times 2$ SOAs for a total of 384 criterion trials per participant. Variable combinations were presented in a different random order to each participant.

\section{Results and Discussion}

Mean proportion of correct responses on valid and invalid trials for each distractor type appears in Figure 5. An omnibus analysis of data of all three groups indicated significant main effects of distractor type $[M$ (small plus $)=.49, M($ skinny vertical $)=.54, M($ vertical $)=.59$, $\left.\chi^{2}(2)=172.81, p<.0001\right]$, SOA $[M(100 \mathrm{msec})=.52, M$ $\left.(200 \mathrm{msec})=.56, \chi^{2}(1)=30.64, p<.0001\right]$, and validity $\left[M(\right.$ invalid $)=.48, M($ valid $)=.74, \chi^{2}(1)=1,253.03$, $p<.0001]$. These effects were qualified by significant interactions of distractor type with SOA $\left[\chi^{2}(2)=6.58\right.$, $p=.0373]$ and with validity $\left[\chi^{2}(2)=74.86, p<.0001\right]$. The validity effect was greatest with the small plus distractor (valid minus invalid difference $=.34$ ), then the skinny vertical (difference $=.27)$, and least with the vertical line distractor (difference $=.17$ ). This difference was due to the differences between distractor types on invalid trials, because there was no significant difference on valid trials as a function of distractor type $\left[\chi^{2}(2)=\right.$ 


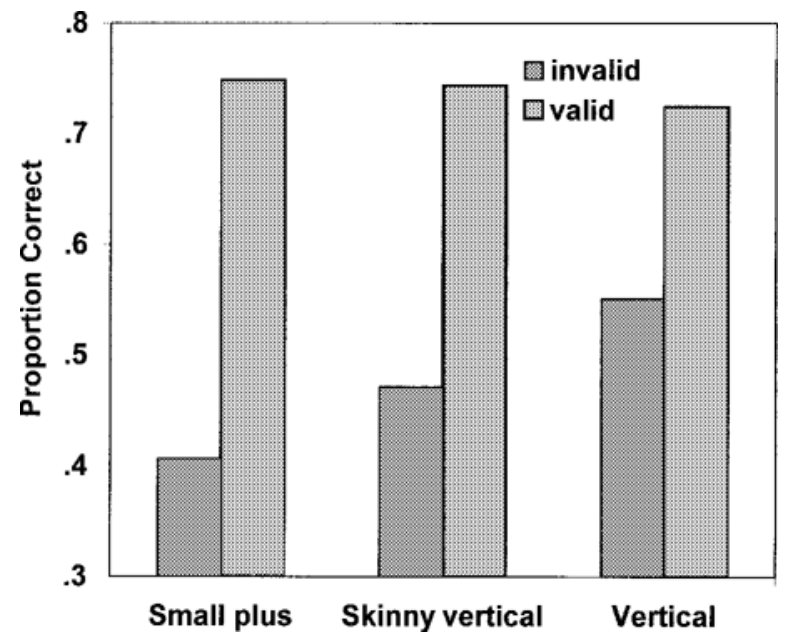

Figure 5. Mean proportion of correct responses on invalid and valid trials for each distractor type in Experiment 2.

$3.14, p=.21]$. Therefore, it appears that all of these distractor types captured attention, but when that attention was directed to a nontarget location, the small plus had more disruptive effects than did either of the other distractors. Although no one commented on it, it is possible that a zooming effect from the original plus to the smaller one made this distractor more salient. The SOA $\times$ validity effect $\left[\chi^{2}(1)=12.14, p<.001\right]$ only showed that the validity effect decreased from .29 (valid proportion correct minus invalid proportion correct) at the $100-\mathrm{msec}$ SOA to .23 at the $200-\mathrm{msec}$ SOA, which suggests that facilitation was decreasing at this interval. The decrease in the validity effects with increase in time between distractors and targets argues against any possible difference between distractors as due to differences in forward masking. Masking effects should result in an increase in validity with increase in SOA. Higher order interactions were not significant.

In this experiment, as in one condition of Atchley et al. (2000), all targets and nontarget characters on the target screen were onsets. Therefore, a screen-wide set for onset could be made. However, there was never a condition to support a set for offsets. In spite of this, there was significant capture by the offset distractor (the vertical line).

Furthermore, the logic of Experiment 2 was that a plus sign changing to a vertical line (the offset) might be considered a change to a new object. However, neither a plus sign that changed to a smaller plus sign nor a plus sign in which the vertical line became thinner necessarily constituted a change to a new object, although it might be argued that a different-shaped plus sign is a different object. In any case, each type captured attention with the least capture by the distractor that was most obviously a new object (the vertical line).

Of course, it is possible to think of each unique distractor as being a singleton and that a set for the singleton $\mathrm{C}$ was made throughout the block of trials. However, even the concept of singleton detection mode does not explain why there should be differences in the amount of capture for the three different distractors. Each distractor type changed by the same number of pixels, but there were still differences between the amount of capture for the three types. This might seem to argue against a hypothesis of a set for a luminance change. However, to make any definitive statement on luminance, it would be necessary to test with equiluminant stimuli.

These data may be interpreted as indicating that offsets do not capture attention because they involve a change to a new object, but instead because the offset itself captures attention. Further support for this interpretation was provided by Experiment 3, in which there was no actual offset, but in which a distractor was provided by the dimming of one of the characters on the fixation screen.

\section{EXPERIMENT 3}

Experiment 1 showed that capture by offset targets might not depend on a specific attentional set, and Experiment 2 suggested that capture from changes in size/ shape are due to the change itself rather than a new object's being produced. Experiment 3 provided further evidence in this respect. The fixation screen contained four bright green squares, one of which dimmed to produce the distractor. The same pixels were illuminated, although more dimly, in the distractor square as in the same square on the fixation screen. Thus, as with the onsets and offsets in Experiments 1 and 2, changes were made to the total luminance of the distractor. In this case, no new object was produced, and capture indicated that a new object was not necessary for attention to be captured by a luminance decrement. As in Experiment 2, one group received a $\mathrm{C}$ among complete circles on the target screen. Because a singleton $\mathrm{C}$ among circles might create a singleton detection mode, a second group received an $\mathrm{E}$ or $\mathrm{H}$ target among $\mathrm{P}, \mathrm{S}$, and $\mathrm{b}$ on the target screen as in Experiment 1 . Thus, there were always four different letters and no singleton on the target screen. This group was used in order to eliminate an explanation of the effects involving a singleton detection strategy (Bacon \& Egeth, 1994).

\section{Method}

There were two groups of 20 participants. One group (6 men and 14 women, mean age of 21.1 years; range of 18-35) received Cs among circles as the targets, presented for $67 \mathrm{msec}$, whereas the other group ( 8 men and 12 women, mean age of 20.9 years; range of 17-33) received $\mathrm{E}$ and $\mathrm{H}$ targets among $\mathrm{P}, \mathrm{S}$, and $\mathrm{b}$ nontargets, presented for $83 \mathrm{msec}$. The exposure duration was made slightly shorter for the Cs among circles in an attempt to produce roughly equal overall performance, relative to the range between chance and perfect accuracy, for the two target types. Pilot work showed that, at a given exposure duration, it seemed to have been easier for participants to locate the $\mathrm{C}$ target among the identical nontarget circles than for participants in the other group to locate the letter target among the different nontarget letters. Poorer performance for $\mathrm{C}$ targets than for $\mathrm{E} / \mathrm{H}$ targets was undoubtedly due to the four-alternative forced choice for Cs and two-alternative forced choice for $\mathrm{E}$ or $\mathrm{H}$. All characters on the target screen were sudden onsets.

For this experiment, the distractor and target locations were arranged as the vertices of a diamond centered on fixation as in Ex- 


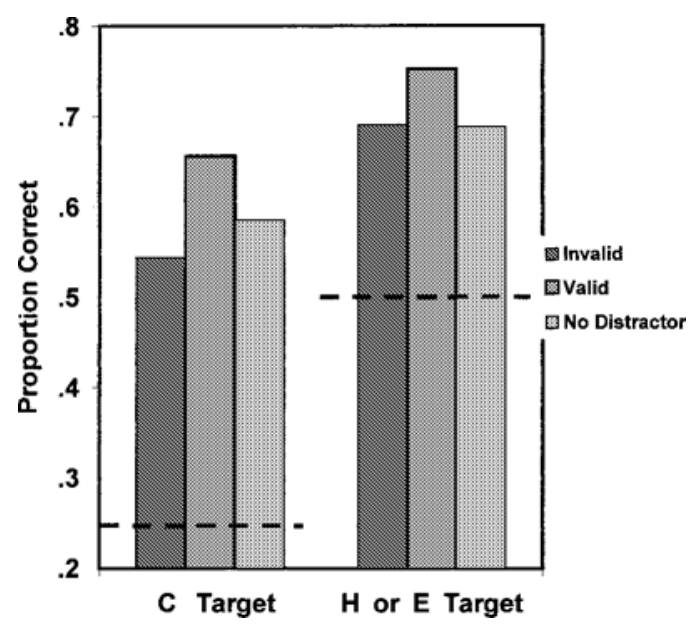

Figure 6. Mean proportion of correct responses for invalid, valid, and no-distractor trials for $\mathrm{C}$ targets and for $\mathrm{H}$ or $\mathrm{E}$ targets in Experiment 3. Dashed lines indicate chance level for each target type.

periment 2. The four locations were the same as in the previous experiment, but on fixation screens these locations contained four bright green squares (IBM color 10, $149.7 \mathrm{~cd} / \mathrm{m}^{2}$ ), each approximately $.72^{\circ}$ tall and wide. One of these changed to dim green (IBM color $2,54.8 \mathrm{~cd} / \mathrm{m} 2)$ to provide the distractor. The targets and masks were bright white (IBM color $15,150.1 \mathrm{~cd} / \mathrm{m}^{2}$ ), on a dark gray background. After 40 practice trials, each participant received 384 criterion trials, which included 96 valid, 216 invalid, and 72 no-distractor (in which all four squares remained bright; no change was made) exposures. The order of all variables was randomized for each participant.

\section{Results and Discussion}

Because chance accuracy differed considerably between circles with gaps (25\%) and $\mathrm{E}$ and $\mathrm{H}$ targets (50\%), data from the two groups were analyzed independently. For the group receiving $\mathrm{C}$ targets (see Figure 6), analysis showed significant main effects of validity $\left[\chi^{2}(2)=\right.$ $57.89, p<.001]$ and SOA $\left[\chi^{2}(1)=27.68, p<.001\right]$. The interaction between validity and SOA was not significant. For the other group, only the same two main effects were significant: validity $\left[\chi^{2}(2)=22.15, p<.001\right]$ and SOA $\left[\chi^{2}(1)=5.87, p=.02\right]$. In the case of the letter targets, there was no singleton to support a singleton detection mode. These results suggest that a simple decrease in luminance captures attention not because it produces a new object or because of a set for luminance decrease (the targets were all onsets, which represent luminance increase), but because of the dimming itself. If a set had been developed on the basis of the targets, it should have been for an increase in luminance, not a decrease in luminance. Both groups showed capture effects: Valid distractors produced significantly higher accuracy than did invalid distractors when the distractor merely dimmed while remaining square in shape. In comparison with the no-distractor condition, accuracy was higher with valid distractors, suggesting benefits of distractors produced by dimming for both target types: for $\mathrm{C}$ targets, $\chi^{2}(1)=$ $57.89, p<.0001$; for E/H targets, $\chi^{2}(1)=16.46, p<$ .0001 .

Similar comparisons between invalid distractors and no distractors showed that there were costs for $\mathrm{C}$ targets $\left[\chi^{2}(1)=9.18, p<.02\right]$ and not for $\mathrm{E} / \mathrm{H}$ targets $\left[\chi^{2}(1)=\right.$ $0.03, p<.86]$. The reason for the difference in the significance of costs between the two target types is not clear, although it is possible that for the $\mathrm{C}$ targets, a singleton detection mode could have increased the capture by the singleton dim square, and/or the more difficult identification could have resulted in more disruption by the invalid distractor.

\section{GENERAL DISCUSSION}

These experiments lead to new interpretations of data in the attentional capture literature. The results show that offset distractors or those that change by shape or luminance may capture attention, and that this capture may not be due to a singleton detection strategy (capture occurred when targets and nontargets all were letters in Experiments 1 and 3 ) or to contingent involuntary orienting caused by an attentional set for targets that share a specific defining characteristic with the distractors (capture occurred with offset distractors and onset targets in Experiments 1 and 2, with onset distractors and offset targets in Experiment 1, and with changes in shape in Experiment 2 and luminance in Experiment 3 with onset targets). The evidence also suggests that capture by change in shape may not be due to the change producing a new visual object. Capture was shown in Experiment 2 when the shape of the distractor character was a plus sign both before and after the change, and in Experiment 3 when the distractor character was produced by dimming rather than by an offset (thus having the same shape and size before and after dimming).

\section{Necessity of Prior Knowledge of Target Type}

These data do not lead to the suggestion that endogenous aspects of visual attention have no effect on whether a stimulus will capture attention. Of course, there are always endogenous aspects of every task, and a change must occur for a target screen to be presented. The present research was planned to address the question of the development of a set for a specific attribute of the target. As stated in the introduction, a set can be developed for a singleton (Bacon \& Egeth, 1994), or a contingent involuntary orienting can occur when there is a contingency between the target and prior distractors (Folk et al., 1993). However, the present data suggest that none of these criteria are necessary for attention to be captured. If there is a salient change on the distractor screen, attention may be captured if there is no overriding attentional set.

\section{Stimuli That Do Not Capture Attention}

A question arises regarding whether attention can be captured with any discontinuity or change on the dis- 
tractor screen. However, there are data that answer "no" to that question. A unique element that produced a discontinuity in color or luminance on a distractor screen that contained multiple elements did not capture attention when all elements appeared simultaneously and the target was one of several white characters (Chastain \& Cheal, 1998). Furthermore, a single plus sign that remained after three of four plus signs on a fixation screen offset did not capture attention (Chastain \& Cheal, 1999). Thus, it appears that some change at the location of the unique distractor element is necessary for capture to occur. For instance, a change (to a unique brightness) in one of multiple distractor elements will not capture attention if the change occurs near the time of distractorscreen onset, but the same change will capture attention if it occurs $1 \mathrm{sec}$ after distractor-screen onset (Cheal \& Chastain, in press). Therefore it is not true that any type of discontinuity or change will necessarily capture attention; the nature and timing of the change is important in the determination of whether capture will occur.

\section{Amount of Capture}

Capture does not seem to be an all-or-none phenomenon. Of course, the strength of validity effects varies with the probability of the distractor's indicating the target location (Gottlob, Cheal, \& Lyon, 1999), but it also varies with the type of distractor even when all types are irrelevant to the target location (Cheal \& Chastain, in press). Experiment 2 revealed different amounts of capture by the three distractor types, with the most capture (as inferred from the amount of disruption on invalid trials) from the small plus distractor and the least from the vertical line distractor. In a study of the time course of attentional effects with onset and offset distractors, Chastain and Cheal (1999) found capture by their offset distractors very fleeting. For offsets, capture was apparent at only one of four SOAs, whereas capture was apparent at every SOA for onsets. Cheal and Chastain (in press) studied distractors involving a flashing or apparently moving unique element among static/nonflashing or apparently moving background elements and found capture with all types. However, there was more capture with static than with apparently moving background elements, and more capture with apparently moving unique elements than with merely flashing ones.

\section{Comparison with Atchley et al.'s (2000) Data}

In very thorough research, Atchley et al. (2000) concluded that offsets can only capture attention when all the stimuli on the target screen appear as offsets. In the work of Atchley et al., and in Experiment 1 here, the target itself was defined by a visual search for one of two letters presented with three other letters. Thus, the target was not defined by any property other than the identity, and no singleton character appeared on the target screen.

In other respects, there were important differences between these studies. In our Experiment 1, there were two onsets and two offsets on each target screen and the target itself could be either an onset or an offset. We chose target screens with both onsets and offsets so that a screen-wide set could not be developed for either onset or offset. What differed was whether the target was an onset or an offset. In contrast, in the Atchley et al. (2000) paradigm, all characters on the target screen were either onsets or offsets. Thus a screen-wide set for onsets or for offsets could be made, and capture occurred only when distractor and target types matched; when they did not match, capture was not significant. Even when the target types were mixed within blocks (as in their Experiment 2), the type of target was shown at the beginning of the trial. When target type could not be predicted (their Experiment 3), capture was found with both onset and offset distractors and/or targets. Similarly, in the present Experiment 1 , offset distractors captured attention when onset and offset targets were mixed. It is doubtful that the type of target was predicted, given the fact that accuracy was much poorer than in the blocked condition. Also, attention was captured by offset distractors when only onset targets appeared within a block. In the latter case, if an attentional set had developed, it should have been for onsets.

In the present experiment, even though the transience of the target was known in advance (for our blocked group), it is possible that no significant set for onset or offset was made and that the transient nature of either the onset or the offset distractor was sufficient to capture attention. Thus, there appears to be a screen-wide set when all target-screen characters are onset or offset that overrides any underlying stimulus-driven effects. This did not occur with the target screens in which only half of the characters were onsets and half were offsets, and thus the stimulus-driven effects of either onsets or offset could appear.

Another possible difference between our Experiment 1 and that of Atchley et al. (2000) was that their distractor screens consisted of four boxes at the four possible target locations. These boxes could contain a figure 8 for target offset trials. In addition, each box could have a small dot above, below, and to the left and right. The onset or offset of the dots around one of the boxes constituted the onset or offset distractor, respectively, Thus, in comparison with the total amount of figural information in the display, the onset or offset of distractors was rather subtle, and perhaps participants had to be actively seeking the corresponding target type in order to notice it. In contrast, there were no boxes around potential target locations in the present experiments. The preview characters in Experiments 1 and 2 were complete plus signs, among which the change to distractors might have been more salient than the onset or offset of dots used by Atchley et al. In our Experiment 3, the distractor characters were bright squares on the perimeter of an imaginary circle around fixation (and nearer fixation than the target locations), among which one dimming may have been quite noticeable. Therefore, even with onset targets, the change produced by distractor offset or dimming was substantial enough to be apparent and therefore to capture attention. In the case where the luminance change 
was minimal, capture did not occur (Experiment 4A, Atchley et al., 2000). Of course, a set for a particular type of stimulus may override the saliency of a particular stimulus, as has been shown previously (Atchley et al., 2000; Bacon \& Egeth, 1994; Folk et al., 1992; Folk et al., 1994; Gibson \& Kelsey, 1998).

\section{Salience}

A possible explanation for many differences in capture involves saliency. Theeuwes (1992) concluded that "selectivity depends on the relative discriminability of the stimulus dimensions" (p. 599)—in other words, on the salience of the character. Although it was shown later that "goal-directed selection of a specific known featural identity may override stimulus-driven capture caused by salient featural singletons" (Bacon \& Egeth, 1994, p. 493), salience may still be very important in certain circumstances.

Thus, a more salient distractor may be more likely to capture attention than a less salient one. With accuracy data, it is debatable whether a more salient distractor captures attention more strongly on each exposure or with equal strength but on a greater proportion of exposures than does a less salient distractor. The finding that attention seems to be captured at longer intervals (across a greater range of SOAs) with onset than with offset distractors (Chastain \& Cheal, 1999) might be interpreted as evidence for the former.

At any rate, salience cannot be the only factor involved, because a distractor element that is very salient owing to its unique color, orientation, or brightness nevertheless does not capture attention if it appears simultaneously with similar background elements (Chastain, 1996; Chastain \& Cheal, 1998; Cheal \& Chastain, in press). It is possible that the success of salience in the capture of attention may vary with several other parameters of the task. For instance, saliency itself may be dependent on the surrounding elements. Changes to existing objects may make them particularly salient. An illustration of this may have been the capture by the dimming of a square in Experiment 3. Resolution of this issue awaits further research.

\section{Attentional Set}

Capture was found when distractor and target types did not match (i.e., one an offset, change in feature, or decrease in luminance, and the other an onset) in all three experiments reported here. Thus, although attentional capture is more apt to occur when a set has been formed for a particular type of target (Atchley et al., 2000), capture may also occur in the absence of a specific set. This was shown in the present experiments and by Experiment 3 of Atchley et al. (2000) when onset and offset distractors and targets were mixed within blocks and no clues were given as to which type would occur. It also was shown when no new object was formed but the distractor changed size, thickness, or luminance and when targets were all onsets (Experiments 2 and 3 ).

\section{Conclusions}

These experiments show that offset distractors may capture attention even when the participants are prepared for an onset target. Also, this capture was not due to a singleton detection strategy, because capture was found when the distractor was a singleton but the target was one of four different letters on the screen. And, in addition, the capture by offsets was not due to the offsets' producing a new visual object. This was shown when the shape of the distractor did not change, but merely changed in luminance. These data support the hypothesis that capture of attention depends on a number of factors in the procedure. Some of these factors include the saliency of the capturing distractor, the type of target, the other characters on the target screen, the timing of the displays, and prior information about the target and displays.

\section{REFERENCES}

Atchley, P., Kramer, A. F., \& Hillstrom, A. P. (2000). Contingent capture for onsets and offsets: Attentional set for perceptual transients. Journal of Experimental Psychology: Human Perception \& Performance, 26, 594-606.

BAcon, W. F., \& Egeth, H. E. (1994). Overriding stimulus-driven attentional capture. Perception \& Psychophysics, 55, 485-496.

Chastain, G. (1996). Multiple-element line segment precues: Orientation and location effects on attention. Perception \& Psychophysics, 58, 1015-1025.

Chastain, G., \& Cheal, M. (1998). Automatic versus directed attention with single-element and multiple-element precues. Visual Cognition, 5, 339-364.

Chastain, G., \& Cheal, M. (1999). Time course of attention effects with abrupt-onset and offset single- and multiple-element precues. American Journal of Psychology, 112, 411-436.

Chastain, G., \& Cheal, M. (2001). Timing offacilitatory and inhibitory effects of visual attention. Manuscript submitted for publication.

Chastain, G., Cheal, M., \& Kuskova, V. (in press). Inappropriate capture by diversionary dynamic elements. Visual Cognition.

Cheal, M., \& Chastain, G. (in press). Allocation of visual attention depends on type of precue. Genetic, Social, \& General Psychology Monographs.

Cheal, M., \& Lyon, D. (1989). Attention effects on form discrimination at different eccentricities. Quarterly Journal of Experimental Psychology, 41A, 719-746.

ERIKSEn, C. W., \& Collins, J. F. (1969). Temporal course of selective attention. Journal of Experimental Psychology, 80, 254-261.

ERIKSEN, C. W., \& Hoffman, J. E. (1972a). Some characteristics of selective attention in visual perception determined by vocal reaction time. Perception \& Psychophysics, 11, 169-171.

ERIKSEN, C. W., \& Hoffman, J. E. (1972b). Temporal and spatial characteristics of selective encoding from visual displays. Perception \& Psychophysics, 12, 201-204.

Eriksen, C. W., \& Hoffman, J. E. (1973). The extent of processing of noise elements during selective encoding from visual displays. Perception \& Psychophysics, 14, 155-160.

Folk, C. L., Remington, R. W., \& Johnston, J. C. (1992). Involuntary covert orienting is contingent on attentional control settings. Journal of Experimental Psychology: Human Perception \& Performance, 18, 1030-1044.

Folk, C. L., Remington, R. W., \& Johnston, J. C. (1993). Contingent attentional capture: A reply to Yantis (1993). Journal of Experimental Psychology: Human Perception \& Performance, 19, 682-685.

Folk, C. L., Remington, R. W., \& Wright, J. H. (1994). The structure of attentional control: Contingent attentional capture by apparent motion, abrupt onset, and color. Journal of Experimental Psychology: Human Perception \& Performance, 20, 317-329.

Gibson, B. S., \& KeLSEY, E. M. (1998). Stimulus driven attentional cap- 
ture is contingent on attentional set for display-wide features. Journal of Experimental Psychology: Human Perception \& Performance, 24, 699-706.

Gottlob, L. R., Cheal, M., \& Lyon, D. R. (1999). Time course of location cuing effects with a probability manipulation. Journal of General Psychology, 126, 261-270.

Hillstrom, A. P., \& YANTis, S. (1994). Visual motion and attentional capture. Perception \& Psychophysics, 55, 399-411.

Holmgren, J. E. (1974). The effect of a visual indicator on rate of visual search: Evidence for processing control. Perception \& Psychophysics, 15, 544-550.

JonIDES, J. (1980). Toward a model of the mind's eye movement. Canadian Journal of Psychology, 34, 103-112.

Jonides, J., \& YANTIS, S. (1988). Uniqueness of abrupt visual onset in capturing attention. Perception \& Psychophysics, 43, 346-354.

Miller, J. (1989). The control of attention by abrupt visual onsets and offsets. Perception \& Psychophysics, 45, 567-571.

Posner, M. I. (1980). Orienting of attention. Quarterly Journal of Experimental Psychology, 32, 3-25.

SPerling, G., \& Melchner, M. (1978). The attention operating characteristic: Examples from visual search. Science, 202, 315-318.
Theeuwes, J. (1990). Perceptual selectivity is task dependent: Evidence from selective search. Acta Psychologica, 74, 81-99.

Theeuwes, J. (1991). Cross-dimensional perceptual selectivity. Perception \& Psychophysics, 50, 184-193.

Theeuwes, J. (1992). Perceptual selectivity for color and form. Perception \& Psychophysics, 51, 599-606.

Watson, D. G., \& Humphreys, G. W. (1995). Automatic capture by contour onsets and offsets: No special role for onsets. Perception \& Psychophysics, 57, 583-597.

Yantis, S. (1993). Stimulus-driven attentional capture. Current Directions in Psychological Science, 2, 156-161.

Yantis, S., \& Hillstrom, A. P. (1994). Stimulus-driven attentional capture: Evidence from equiluminant visual objects. Journal of Experimental Psychology: Human Perception \& Performance, 20, 95-107.

YANTIS, S., \& Jonides, S. (1984). Abrupt visual onsets and selective attention: Evidence from visual search. Journal of Experimental Psychology: Human Perception \& Performance, 10, 601-621.

(Manuscript received May 8, 2000;

revision accepted for publication November 19, 2000.) 